\title{
A importância do diagnóstico precoce no Tratamento do câncer de orofaringe: Estudo de caso.
}

\section{The important of early diagnosis in the tratment of in thetreatment of oropharynx neoplasm: A case study.}

André Luiz Barbosa Santos ${ }^{1}$, Jamil Elias Dib², Leonardo Begalles de Souza ${ }^{3 .}$

\section{RESUMO}

O diagnóstico precoce é uma medida de prevenção secundária que proporciona melhoras nas chances de cura, garante maior sobrevida, aumenta a qualidade de vida e oferece terapias menos mutiladoras em relação a muitos tipos de câncer, dentre eles, o carcinoma espinocelular (CEC). O objetivo do artigo foi estudar o caso de um tumor diagnosticado precocemente como CEC, localizado na região de palato mole, na clínica de odontologia da Universidade de Gurupi - UNIRG, no ano de 2018. A metodologia utilizada foi através de um estudo transversal, fundamentado na análise retrospectiva documental do prontuário do paciente. Diante dos procedimentos realizados, a biópsia excisional teve a sua indicação precisa em se tratando do tamanho do tumor e o laudo inserido do discorrer do caso clínico confirmou que as margens de segurança tanto laterais quanto em profundidades estavam livres de infiltração. Com isso, foi possível mostrar que o diagnóstico precoce proporcionou melhor forma de tratamento para o paciente e que o resultado obtido até o presente momento melhorou a qualidade de vida do mesmo. O tratamento pôde ser efetuado perante uma terapia menos agressiva e que isso poderá repercutir em sua sobrevida global.

Palavras-chave: Diagnóstico precoce. Carcinoma espinocelular. Orofaringe. Palato mole. Tratamento.

\section{ABSTRACT}

Early diagnosis is a secondary preventive step, which increases the likely of cure and thus, guarantees longer patient's survival, increasing quality of life and offering subsequent less invasive and destructive forms of therapy when we consider many types of neoplasms, among them, espino-cellular carcinoma,

(ECC). The goal of this study was to assess and study the case diagnosed early as espino-cellular carcinoma in the region of the soft palate, in the dental clinics School of Dentistry, University of Gurupi-UNIRG, one day in 2018. The method used in this case was cross - sectional based on a retrospective analysis of the patient's chart. An excisional biopsy was indicated based primarily in the neoplasm size and the enclosed report confirmed that safety margins both laterally and in depth were free of infiltration. Consequently, it was possible to confirm that early diagnosis provided the best method of treatment for the patient and the favorable outcome improved his quality of life. Treatment can be carried out using less aggressive therapy which may lead to a longer patient's survival.

Keywords: Early Diagnosis. Espino-Cellular Carcinoma. Oropharynx. Soft Palate. Treatment.
1.Bacharel em Odontologia, Universidade de Gurupi

2. Prof. Adjunto UNIRG. Gurupi -TO. Cirurgião Buco Maxilo Facial. Hospital de Urgências de Goiânia HUGO. Goiânia--Go. Coordenador do Serviço de Cirurgia e Traumatologia Buco Maxilo Facial do Hospital de Urgências de Anápolis - HUANA. Anápolis GO.

Bacharel em Odontologia, Universidade de Gurupi 


\section{INTRODUÇÃO}

O diagnóstico precoce é uma forma de prevenção secundária contra o câncer de boca1. As lesões em estágios iniciais restritas ao sítio primário asseguram melhor sobrevida, maior qualidade de vida e oferecem terapias menos mutiladoras em relação ao tratamento da doença $a^{2,3,4}$.

A maioria dos cânceres orais são diagnosticados tardiamente e vários fatores estão envolvidos para que esse problema aconteça, de modo que o conhecimento ampliado acerca dos fatores de risco assim como das lesões pré-malignas auxilia no diagnóstico inicial das neoplasias malignas da cavidade oral ${ }^{2,1,5,6}$.

O tipo histológico mais comum na cavidade oral é o carcinoma espinocelular (CEC) que está presente em mais de $90 \%$ dos casos de câncer de boca e estes podem surgir em várias regiões da orofaringe principalmente o palato mole e, por isso, sendo incluídos também como neoplasias de cavidade oral, mesmo sabendo que os tumores localizados nessas estruturas apresentam características agressivas e com rápido início de ação ${ }^{7,8,9}$.

No Brasil, a segunda causa de morte dentre as doenças crônico-degenerativas é o câncer, e no ano de 2015 o câncer de boca levou a morte de 5.898 pessoas, destes 4.672 eram do gênero masculino. Para o ano de 2040 estima-se em torno de 545.396 novos casos de neoplasias de lábio e cavidade oral e 142.797 para tumores de orofaringe $\mathrm{e}^{6,10,11}$.

Diante do que foi visto na literatura, o diagnóstico precoce do câncer de boca é fundamental para a cura e qualidade de vida dos pacientes e isso pôde ser comprovado no referido relato de caso.

O objetivo do artigo foi relatar o caso de um tumor diagnosticado precocemente como CEC, localizado na região de palato mole, na clínica de odontologia da Universidade de Gurupi - UNIRG, no ano de 2018.

\section{ESTUDO DE CASO}

O estudo foi transversal e descritivo a partir de uma análise documental retrospectiva do prontuário médico do paciente e teve sua aprovação pelo Comitê de Ética em Pesquisa sob o número 3.563.438, aprovado em 09 de setembro de 2019.

Paciente G.M de 53 anos, pardo, tabagista, gênero masculino, comerciário, natural de Gurupi (TO), compareceu ao ambulatório de patologia oral da Universidade de Gurupi 
(UNIRG), queixando-se de uma mancha vermelha no "céu da boca" sem sintomatologia dolorosa. O paciente negou ser etilista ou usar quaisquer tipos de drogas ilícitas.

À oroscopia, verificou-se uma lesão exantematosa plana, de base séssil, com bordas irregulares e não apresentava ulceração central, localizada na parte anterior do palato mole (figura 1). Ao se realizar a palpação, o paciente não relatou dor e não foi notado sangramento local, porém, de uma consistência endurecida, não apresentando limites e com sinais de fixação aos arredores. Os dentes apresentavam-se com cálculo em todos os sextantes e alguns com mobilidade grau 2 e 3 . À ectoscopia não revelou linfonodos submandibulares ou cervicais palpáveis ou qualquer outro tipo de alteração. A radiografia digital panorâmica, póstero-anterior e perfil do tórax não apontaram nenhum achado relevante (figura 2 e 3 ).

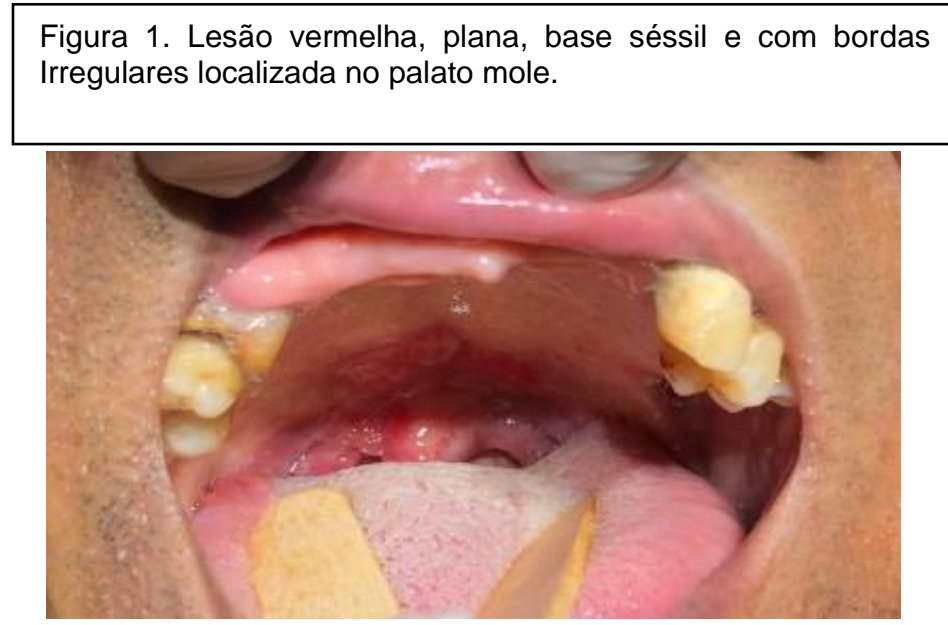

Figura 2. Radiografia digital de tórax apresentando padrões de normalidade em suas estruturas.

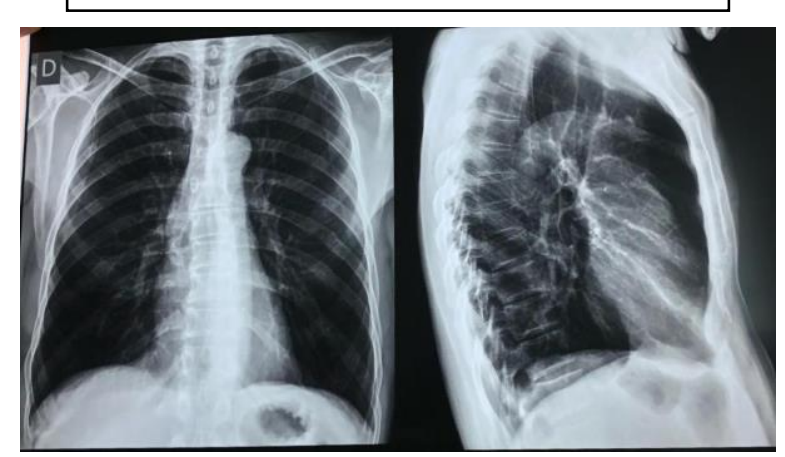

Figura 3. Radiografia digital panorâmica.

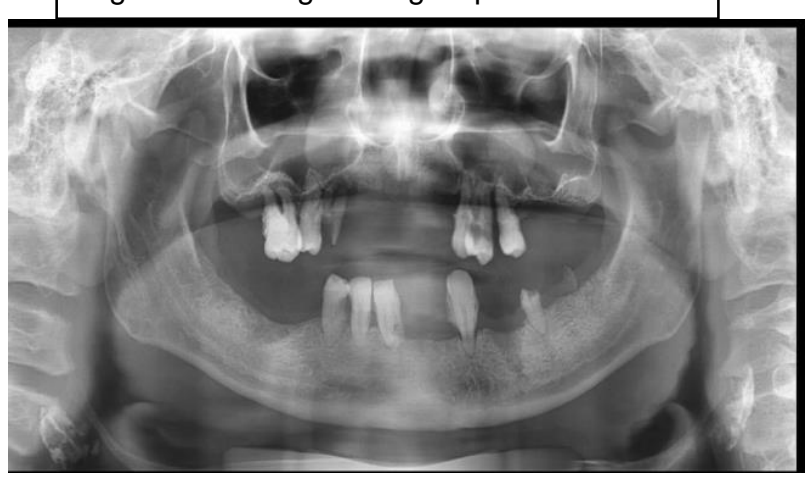


Foi realizada uma biópsia do tipo excisional (20-09-2018), visando obter as margens de segurança (figura 4), e o material coletado foi acondicionado em formol a $10 \%$ e enviado para a análise histopatológica. A macroscopia foi descrita como material medindo $2,0 \times 1,5 \times 0,6 \mathrm{~cm}$. A descrição microscópica mostrou uma lesão representada por um tecido epitelial estratificado paracetótico atrófico, com vários graus de displasia, com área de infiltração desordenada de células epiteliais para o interior do tecido conjuntivo. As células neoplásicas apresentavam formas variadas, com hipercromatismo nuclear e elevado número de mitoses por campo. Estas invadiam o tecido subjacente rico em glândulas mucosecretoras, sialodenite, sialodoquite e com formação de cisto de retenção. O diagnóstico obtido foi Carcinoma Escamoso moderadamente diferenciado (figura 5).

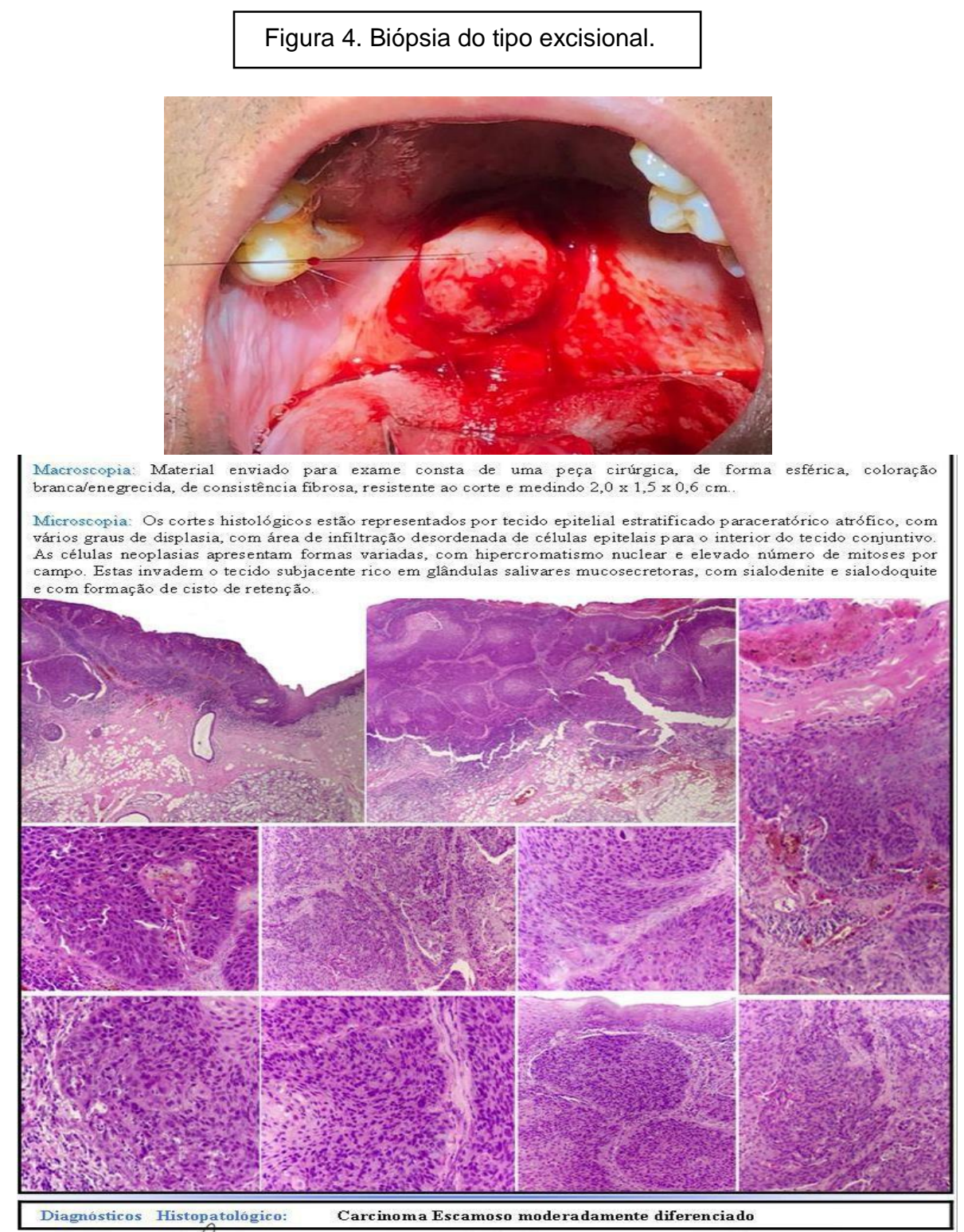


O paciente foi encaminhado para o centro de especialidades odontológicas (CEO) e em seguida seguiu para o Hospital Geral de Palmas - TO (HGP) e lá o tumor foi classificado como sendo T2 N0 M0 e foi programado o tratamento radioterápico.

Antes do início da terapia, o meio bucal do paciente foi adequado, e assim todos os seus dentes foram extraídos por não apresentarem um bom nível de inserção clínica devido a doença periodontal avançada.

Foram realizadas 35 sessões de radioterapia de intensidade modulada (IMRT) com dose diária de 2 Gy em um total de 70 unidade Gray.

Foi instituído o uso de chá de camomila gelado durante o período das terapias e até o fim do tratamento. Além disso, a odontologia oncológica ficou responsável pela laserterapia, e usou o laser de baixa intensidade de $100 \mathrm{~mW}$ de potência, com comprimento de onda de $660 \mathrm{~nm}$ e $4 \mathrm{~J} / \mathrm{cm}^{2}$ que foi aplicado 3 vezes por semana em dias alternados com o objetivo de prevenir mucosite e xerostomia.

O paciente só teve condições de iniciar a confecção da prótese total 6 meses depois de finalizar a radioterapia.

Atualmente o paciente encontra-se bem e sem resquícios da doença de acordo com as avaliações realizadas pelas equipes de oncologia e da patologia oral e maxilofacial, não houve a necessidade de cirurgias ressectivas, doses de radioterapia exaustivas e também não houve indicação para a quimioterapia (figura 6).

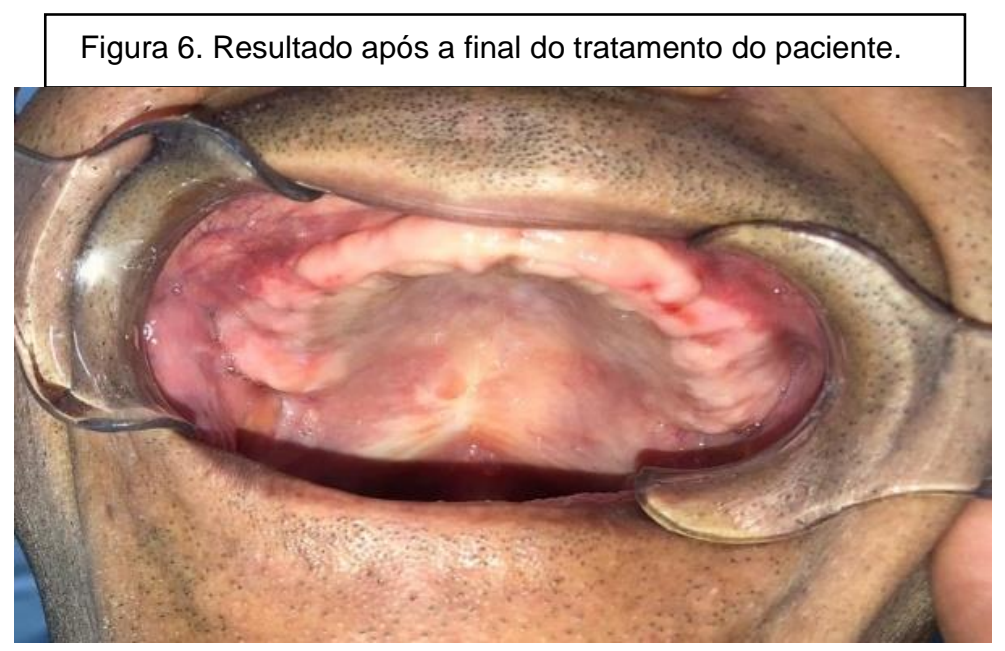

\section{DISCUSSÄO}

O diagnóstico do CEC baseia-se não apenas em evidências clínicas, mas na sua comprovação através de biópsias ${ }^{12}$ e 0 exame histopatológico é imprescindível para 
confirmação do tipo de tumor e descartar ou não sua malignidade ${ }^{13}$. Estudos ${ }^{14}$ mostram a supremacia da biópsia excisional em relação a biópsia incisional em decorrência da maioria das lesões serem pequenas, o que vem de encontro com o referido estudo de caso onde a biópsia de escolha foi a excisional, levando em consideração o tamanho da lesão.

A neoplasia referenciada neste trabalho foi encontrada na parte anterior do palato mole estendendo-se até próximo da úvula. Apesar de ser considerada rara ${ }^{9}$, um trabalho ${ }^{5}$ mostrou que o palato mole foi a segunda região anatômica mais frequente para o câncer de boca.

O resultado obtido através do exame histopatológico permite chegar ao diagnóstico definitivo de carcinoma escamoso moderadamente diferenciado e a lesão foi classificada como T2 N0 M0. A classificação TNM tem maior ligação com o prognóstico do que a classificação microscópica ${ }^{8}$, mas os dados obtidos em outro estudo ${ }^{15}$ conferem uma relação estatisticamente significativa da classificação microscópica com os estadiamento clínico TNM. Os tumores localizados na região do palato mole possuem características agressivas e com rápido início de ação e podem apresentar metástases mesmo para lesões primárias ${ }^{9}$, o que difere do caso aqui estudado onde não apresentou metástases cervicais nem a distância.

A radioterapia na região de cabeça e pescoço promove alguns efeitos adversos na cavidade oral, de modo que, antes do início do tratamento, deverá ser feita a adequação do meio dentre eles, a remoção da raízes residuais e os dentes que se encontrarem com problemas periodontais graves devem ser extraídos, e as cáries deverão ser tratadas ${ }^{16}$. A radioterapia por si só causa modificações no alvéolo, ampliação do ligamento periodontal e destruição do osso trabecular, então os dentes com maior comprometimento periodontal devem ser removidos antes do início da terapia ${ }^{17}$. O paciente citado neste estudo apresentava grandes perdas de inserção clínica e os dentes com muito acúmulo de cálculo, portanto, todos eles foram extraídos com objetivo de eliminar os focos infecciosos e prevenir sequelas pós radioterapia, dentre elas, a osteorradionecrose.

As taxas de sobrevida para tumores de T1 (91 a 100\%) e T2 (70 a 75\%) são altas em comparação a estágios T3 e T4 ${ }^{9}$. O estadiamento clínico também auxilia no tratamento do tumor e aqueles sediados no palato mole podem ser tratados por meio da cirurgia mas elas podem promover a diminuição na qualidade de vida do paciente, devido a sequelas deixadas por tal procedimento (dificuldade para falar e deglutir) ${ }^{3}$. No entanto 
para tumores em estágios iniciais na região do palato mole a cirurgia é a melhor opção e favorece na sobrevida global do paciente ${ }^{9}$, e foi adotada no caso em questão em que a própria biopsia excisional revelou margens de segurança tanto nas laterais quanto em profundidade.

Outra estratégia é abordada na literatura ${ }^{18}$ através das quais acreditam que a melhor opção de tratamento para neoplasias em palato mole é a IMRT seguida ou não de braquiterapia de altas taxas de dose. Nesse estudo de caso aqui apresentado, a IMRT foi o tratamento empregado. Essa forma de tratamento permite a utilização da irradiação de forma mais concentrada e intensa além de preservar os tecidos vizinhos por se tratar de um tratamento mais seletivo ao tumor ${ }^{8,46}$. Os tumores em estágios avançados apresentam tratamentos mais radicais que incluem cirurgias extensas, radioterapia com doses exaustivas e quimioterapia, que são empregadas de formas isoladas ou concomitantes 2,7,8,19, o que não foi necessário ao caso aqui relatado.

A laserterapia pode ser empregada durante e depois do tratamento radioterápico com o objetivo de prevenir e tratar mucosite e xerostomia ${ }^{17}$ e esse fato está em concordância com o estudo aqui abordado pois também foi empregado esta medida pelo Cirurgião Dentista $(C D)$, mostrando assim a importância do $C D$ oncológico na equipe multidisciplinar antes, durante e depois do tratamento antineoplásico ${ }^{20}$. Neste presente estudo de caso também foi empregado o bochecho com chá de camomila gelado, que apresenta propriedades anti-inflamatórias, atua na cicatrização de feridas, tem ação bacteriostática e propriedades antissépticas ${ }^{21}$. Essas medidas proporcionaram um conforto durante e no pós-tratamento.

A prótese total do paciente só foi iniciada 6 meses após a radioterapia, o que está em concordância com outro estudo ${ }^{20}$ onde os pacientes não conseguiam utilizar próteses imediatamente após a conclusão da terapia antineoplásica por causa dos diferentes efeitos colaterais presentes (mucosite, edema, xerostomia).

\section{CONSIDERAÇOES FINAIS}

O diagnóstico precoce favorece no tratamento do câncer de orofaringe, promovendo melhor qualidade de vida para o paciente com melhores índices de sobrevida. 
O tratamento precoce evita a necessidade de tratamento cirúrgicos ressectivos, ou mais radicais e também doses exaustivas de radioterapia e nem de abordagem quimioterápica.

Além disso, o diagnóstico e os métodos precoces de tratamento evitam risco de metástases cervicais e a distância neste paciente, mesmo sabendo que são mais suscetíveis na orofaringe.

O Cirurgião Dentista é um instrumento fundamental tanto na identificação da lesão podendo promover o diagnóstico precoce ou ainda pode participar da equipe multidisciplinar antes, durante e depois do tratamento do paciente, no sentido de prevenir e minimizar as complicações e sequelas pós operatórias.

\section{REFERÉNCIAS}

1- Torres-Pereira Cassius C, et al. Abordagem do câncer da boca: uma estratégia para os níveis primário e secundário de atenção em saúde. Cadernos de Saúde Pública. 2012; 28:30-39.

2-Bonfante Gisele Macedo da Silva, et al. Sobrevida de cinco anos e fatores associados ao câncer de boca para pacientes em tratamento oncológico ambulatorial pelo Sistema Único de Saúde. Cadernos de Saúde Pública. 2014; 30:983-997.

3-Chan Carmen K, et al. Squamous Cell Carcinoma of the Soft Palate in the United States: A Population-Based Study. Otolaryngology-Head and Neck Surgery. 2018;159(4):662-668.

4-De Sousa Bárbara Libardi, et al. Conhecimento dos alunos de Odontologia na identificação do câncer oral. Revista Brasileira de Odontologia. 2016;73(3):186.

5-Santos Luiz Carlos Oliveira dos, BATISTA Olívio de Medeiros, CANGUSSU Maria Cristina Teixeira. Caracterização do diagnóstico tardio do câncer de boca no estado de Alagoas. Braz. j. otorhinolaryngol. 2010.

6- Silva João Gabriel, et al. Comportamentos e conhecimentos de cirurgiões-dentistas da atenção primária à saúde quanto ao câncer bucal. Cadernos Saúde Coletiva. 2016.

7-Galbiatti Ana Lívia Silva, et al. Head and neck cancer: causes, prevention and treatment. Brazilian journal of otorhinolaryngology. 2013;79(2):239-247.

8- Neville Brad W, et al. Patologia oral e maxilofacial. Elsevier Brasil; 2016. ISBN: 9788535265644 . 
9-Schernberg Antoine, et al. Prognostic factors in patients with soft palate squamous cell carcinoma. Head \& neck. 2016.

10-Inca-instituto nacional do câncer; 2018. Incidência de câncer no Brasil, Câncer de cavidade oral.; [cited 2019 May 29]; Available from: http://www1.inca.gov.br/estimativa/2018/sintese-de-resultados-comentarios.asp>.

11-Cancer Tomorrow: Cancer Tomorrow [Internet]. IARC-International Agency for Research on Cancer, World Health Organization, GVO; 2018 [cited 2019 May 29]. Available from: https://gco.iarc.fr/tomorrow/graphicisotype?type $=0$ \&population $=900 \&$ mode $=$ population \&sex $=0 \&$ cancer $=39 \&$ age_group=value \&apc_male $=0 \& a p c \_f e m a l e=0$

12-Câncer de boca: INCA-instituto nacional do câncer; 2018 [cited 2020 May 30]. Available from: https://www.inca.gov.br/tipos-de-cancer/cancer-de-boca.

13-Fontes Karla Bianca F. C., et al. Contribuição da citopatologia para o diagnóstico de carcinoma de células escamosas oral. J. Bras. Patol. Med. Lab. 2008;44(1):17-24.

14-Aquino Sibele Nascimento de, et al. Concordância entre diagnóstico clínico e histopatológico de lesões bucais. RGO. Revista Gaúcha de Odontologia. 2010;58(3):345349.

15- Costa Antonio de Lisboa Lopes, et al. Correlação entre a classificação TNM, gradação histológica e localização anatômica em carcinoma epidermóide oral. Pesquisa Odontológica Brasileira. 2002;16(3):216-220.

16-Vieira Danielle Leal, et al. Tratamento odontológico em pacientes oncológicos. Oral Sciences. 2012;37-42.

17-Rolim Ana Emília Holanda, DA COSTA Lino João, RAMALHO Luciana Maria Pedreira. Repercussões da radioterapia na região orofacial e seu tratamento. Radiologia Brasileira. 2011;44(6):388-395.

18- Marta Gustavo Nader, HANNA Samir Abdallah, DA SILVA João Luis Fernades. Carcinoma epidermoide de palato mole: relato de tratamento não convencional com radiação: externa (IMRT) e braquiterapia (HDR). Revista da Faculdade de Ciências Médicas de Sorocaba. 2010;12(4):20-23.

19-Brener Sylvie, et al. Carcinoma de células escamosas bucal: uma revisão de literatura entre o perfil do paciente, estadiamento clínico e tratamento proposto. Rev Bras Cancerol. 2007;53(1):63-69. 
20-Quispe $\mathrm{Ra}$, et al. Estudo caso-controle de índices de doenças bucais em indivíduos com câncer de cabeça e pescoço após terapia antineoplásica. Einstein. 2018;16(3)

21-Schirmer EM, Ferrari A, Trindade LC. Evolução da mucosite oral após intervenção nutricional em pacientes oncológicos no serviço de cuidados paliativos. Rev. dor. 2012;13(2):141-146. 